\title{
A Compact, Passive Frequency-Hopping Harmonic Sensor Based on a Microfluidic Reconfigurable Dual-Band Antenna
}

\author{
Liang Zhu, Mohamed Farhat, Yi-Chao Chen, Khaled N Salama, Senior Member, IEEE, \\ and Pai-Yen Chen, Senior Member, IEEE
}

\begin{abstract}
We propose here a fully-passive wireless liquid sensor using a harmonic transponder, which comprises a dual-band microstrip antenna reconfigured by different types of liquids injected in a fluidic cavity. Different from traditional radiofrequency (RF) backscatter sensors, the proposed harmonictransponder sensor (or harmonic sensor) receives frequencyhopped RF monotones and backscatters their second harmonics, with the peak frequency shifted by dielectric properties of liquid mixtures. This microstrip antenna has a hybrid-feed structure, of which an outer split-ring patch exhibits a narrow-band $\mathbf{T M}_{310}$ mode at the fundamental frequency $\left(f_{0}\right)$ and an inner elliptical patch displays a wideband resonance centered at the second-harmonic frequency $\left(2 f_{0}\right)$, achieved with hybridization of $\mathrm{TM}_{e 110}$ and $\mathbf{T} \mathrm{M}_{o 110}$ modes. In particular, the outer split-ring patch is loaded with a fluidic channel system to tune the resonance frequency of the $T M_{310}$ mode $\left(f_{0}\right)$. We demonstrate that the type of liquid mixture filling in the fluidic cavity can be clearly perceived by reading the peak received signal strength indicator (RSSI) in the spectrum of second harmonics. Our results show the potential for deploying this passive wireless sensor in noisy environments that include clutters, multiple reflections, jamming, and crosstalks.
\end{abstract}

Index Terms - antenna sensors, harmonic-transponder sensors, passive wireless sensors, microstrip antennas, clutters, cross talks, electromagnetic interferences.

\section{INTRODUCTION}

Wireless sensing and tracking are core technologies that enable many applications in the scope of internet-of-things (IoT), industrial 4.0, wireless healthcare, and smart city, to name a few [1]-[4]. In most wireless sensing systems, a reader or interrogator transmits a continuous wave $(\mathrm{CW})$ radiofrequency (RF) signal to power a tag and receives backscattered signals that are modulated by a sensor or actuator on the tag [5][7]. Along with development of ubiquitous massive sensors, the ever-increasing wireless nodes and the combination of heterogeneous networks have already generated a time-varying and rich-scattering complex environment filled with echoes, clutters, crosstalks and other electromagnetic interference sources. Very recently, the concept of harmonic sensor was inspired by the concept of harmonic radar, and has gained considerable attention because it provides a longer detection range and an enhanced signal-to-noise ratio (SNR) for electrically-small passive wireless sensors [8]-[12]. These

L. Zhu and P. Y. Chen are with the Department of Electrical and Computer Engineering, University of Illinois, Chicago, IL 60607, USA.

Y. C. Chen is with the Department of Computer Science and Engineering, Shanghai Jiao Tong University, Shanghai 200240, China

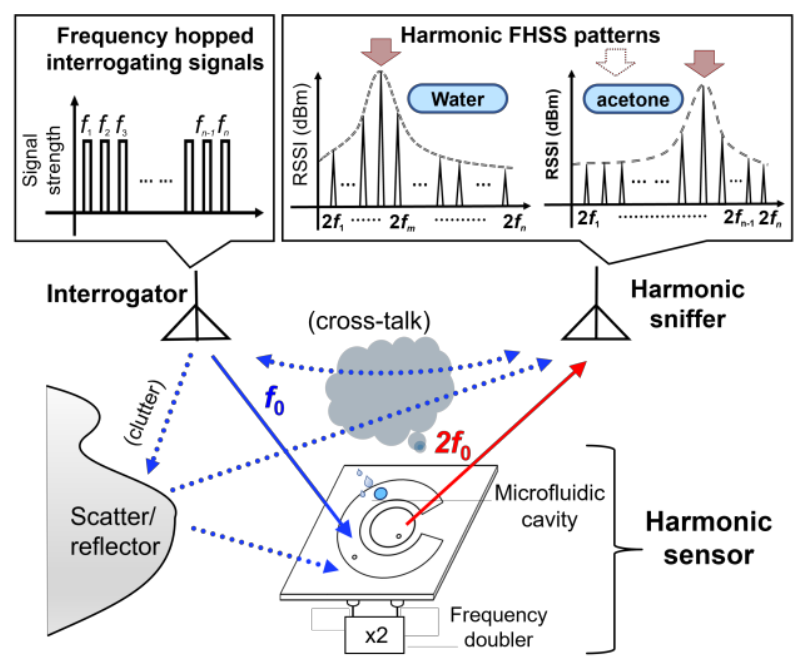

Fig. 1. Schematics and sensing mechanism of a frequency-hopping harmonic sensor based on the proposed dual-band microstrip antenna. The antenna displays a liquid-reconfigured narrow resonance at the fundamental frequency $f_{0}$ and an insensitive wideband resonance centering in the second-harmonic band $2 f_{0}$. The full-passive harmonic sensor receives and backscatters RF signals at orthogonal frequencies to avoid clutters and crosstalks. The types of liquids inside the microfluidic cavity can be known by analyzing the frequency hopping spread spectrum (FHSS) pattern.

harmonic sensors can be classified as nonlinear antenna sensors, whose resonant frequency can be sensitively reconfigured by the dielectric characteristics of the sample under test (SUT) [13]-[16]. Unlike traditional radio-frequency identification (RFID)-based passive sensors, a compact harmonic sensor receives a monotone signal at the fundamental frequency $\left(f_{0}\right)$ and retransmits the modulated high/sub-harmonic signal $\left(n f_{0}\right)$ to monostatic or bistatic readers. By transmitting and detecting RF signals at orthogonal frequencies, this harmonicbased wireless sensing system has the capability to greatly suppress clutters, echo noises, and crosstalks, even though the nonlinear sensor has a small footprint and radar cross-section (RCS). Generally speaking, harmonic sensors have been demonstrated to be effective in several applications, such as tracking small-RCS insects or frogs [17]-[21], RECCO avalanche victim searching system [22], and continuous monitoring of biological parameter, humidity, and temperature monitoring [23]-[27]. Despite these widespread applications,

M. Farhat and K. N. Salama are with the Division of Computer, Electrical, and Mathematical Science and Engineering, King Abdullah University of Science and Technology (KAUST), Thuwal 23955-6900, Saudi Arabia.

This work was supported by NSF ECCS-CCSS under Grant 1914420. Corresponding author: P. Y. Chen; e-mail: pychen@uic.edu 
1

2

3

4

5

6

7

8

9

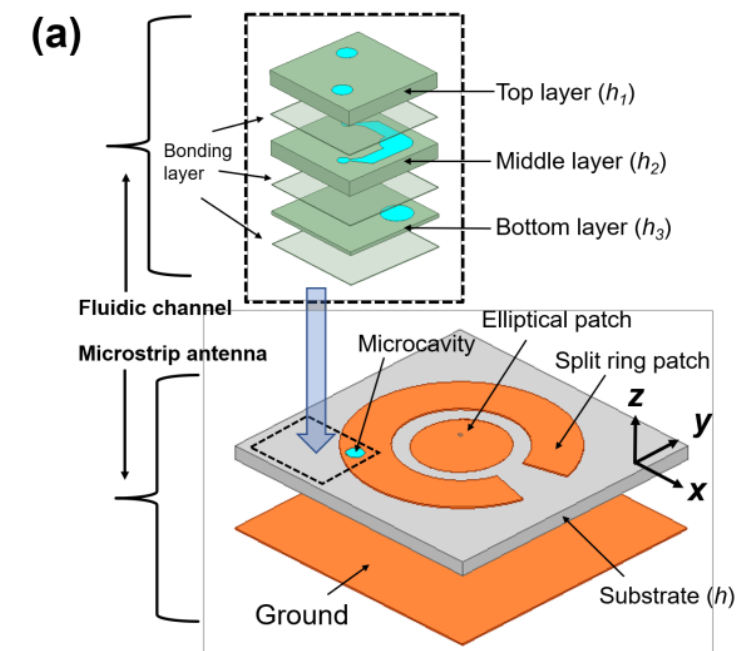

(b)

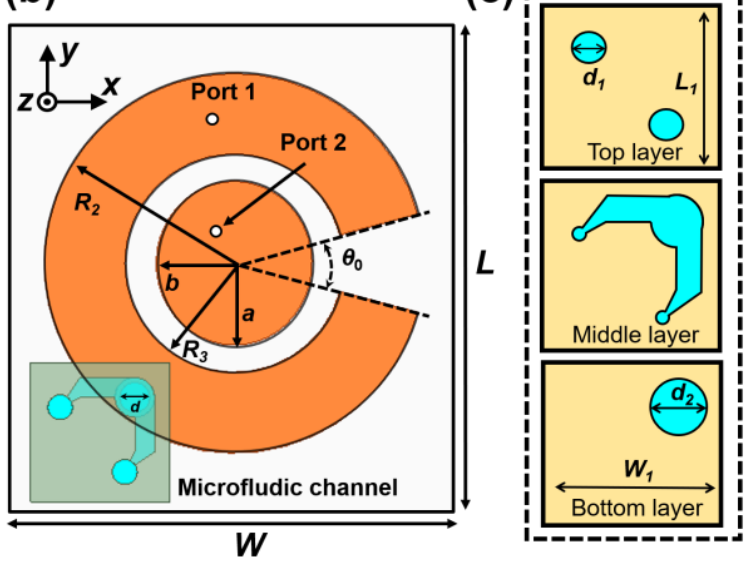

Fig. 2. Geometry and dimensions of the proposed dual band microstrip antenna with fluidic channel: (a) 3-D view. (b) Top view of the microstrip antenna. (c) Top view of the three-layers fluidic channel.

Table I

Dimensions of the proposed antenna in Fig. 2 (Unit: $\mathrm{mm}$ )

\begin{tabular}{|c|c|c|c|c|}
\hline $\boldsymbol{L}=85$ & $\boldsymbol{L}_{\boldsymbol{1}}=22$ & $\boldsymbol{W}=85$ & $\boldsymbol{W}_{\boldsymbol{1}}=22$ & $\boldsymbol{h}_{\boldsymbol{1}}=1.5$ \\
\hline $\boldsymbol{h}_{\boldsymbol{2}}=1.5$ & $\boldsymbol{h}_{3}=0.1$ & $\boldsymbol{a}=16.5$ & $\boldsymbol{b}=15.675$ & $\boldsymbol{\theta}_{\boldsymbol{0}}=30$ \\
\hline $\boldsymbol{R}_{\mathbf{2}}=38.2$ & $\boldsymbol{R}_{3}=22$ & $\boldsymbol{d}=4$ & $\boldsymbol{d}_{\boldsymbol{1}}=3.2$ & $\boldsymbol{d}_{\boldsymbol{2}}=5$ \\
\hline
\end{tabular}

until now, harmonic sensors still suffer from several pitfalls. For example, they generally requires dual antennas, i.e., a $R_{x}$ antenna to receive the fundamental tone $\left(f_{0}\right)$ and a $T_{x}$ antenna to retransmit the harmonic signal (e.g., second harmonic or $2 f_{0}$ ) [28], [29], which inevitably increases the overall sensor/tag size and cost. Besides, wireless readout that relies merely on detecting the amplitude of backscattered second harmonic could still struggle with errors and data misinterpretation due to path loss and reflections of second harmonic. Although the widespectrum absolute resonance sensing could address this issue, microstrip antennas used in traditional RFID tags are usually narrowband [28], [29], and, therefore, not suitable for widespectrum applications.

In this paper, we propose a compact, reconfigurable dualband microstrip antenna that can be used for making a low- (a) $\mathrm{TM}_{310}(1.31 \mathrm{GHz})$

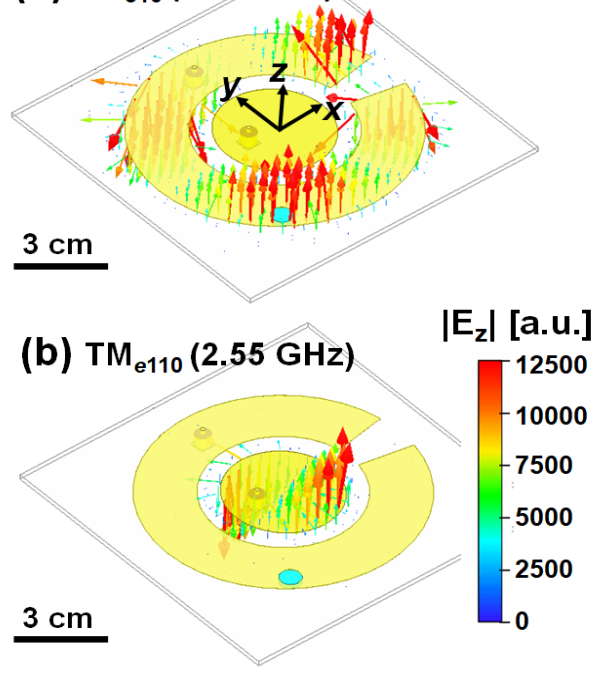

(c) $\mathrm{TM}_{\mathrm{o} 110}(2.64 \mathrm{GHz})$

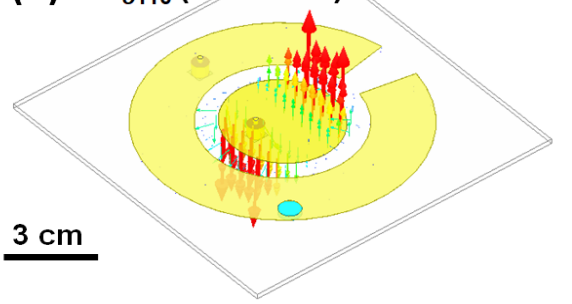

Fig. 3. Simulation results for snapshots of electric field $E_{z}$ : (a) $\mathrm{TM}_{310}$ mode at $1.31 \mathrm{GHz}$, (b) $\mathrm{TM}_{e 110}$ mode at $2.55 \mathrm{GHz}$, and (c) $\mathrm{TM}_{o 110}$ mode at 2.64 $\mathrm{GHz}$.

profile passive harmonic sensor to monitor liquid density in real time. Specifically, this harmonic sensor adopts the robust widespectrum absolute resonance sensing [Fig. 1] that has not yet been explored in existing RFID sensor systems. This antenna excites a narrowband resonant mode at $f_{0}$ and a broadband resonance at $2 f_{0}$, which is necessary for the frequency-hopping harmonics-based sensing. In addition, as understood from the eigenmodal analysis, the microstrip antenna is properly loaded with a fluidic cavity such that perturbations caused by the liquid's dielectric properties can shift the resonance frequency $f_{0}$, while not affecting the broadband resonance around $2 f_{0}$. Consequently, a nonlinear, wide-spectrum absolute resonance sensing scheme can be achieved by analyzing the frequency hopping spread spectrum (FHSS) of the reader, as illustrated in Fig. 1.

In the proposed wireless sensing system, the reader's transceiver $\left(T_{x}\right)$ transmits a constant-strength frequencyhopping sequence with 20 channels $\left[f_{1}, f_{2}, \ldots, f_{20}\right]$ to the harmonic sensor equipped with the proposed dual-band antenna. These hopping signals are received in sequence by the harmonic sensor, undergoing the frequency doubling process $\left[f_{1}, f_{2}, \ldots, f_{20}\right] \rightarrow\left[2 f_{1}, 2 f_{2}, \ldots, 2 f_{20}\right]$, and being re-transmitted to the portable sniffer (e.g. smart phone with 5G and LTE antenna covering the frequency band of interest). The dielectric property of the SUT can effectively modulate the antenna's resonance frequency $f_{0}$, which will be extracted by post-processing the high-dimensional FHSS pattern $\left[2 f_{1}, 2 f_{2} \ldots 2 f_{20}\right]$, as sketched in 

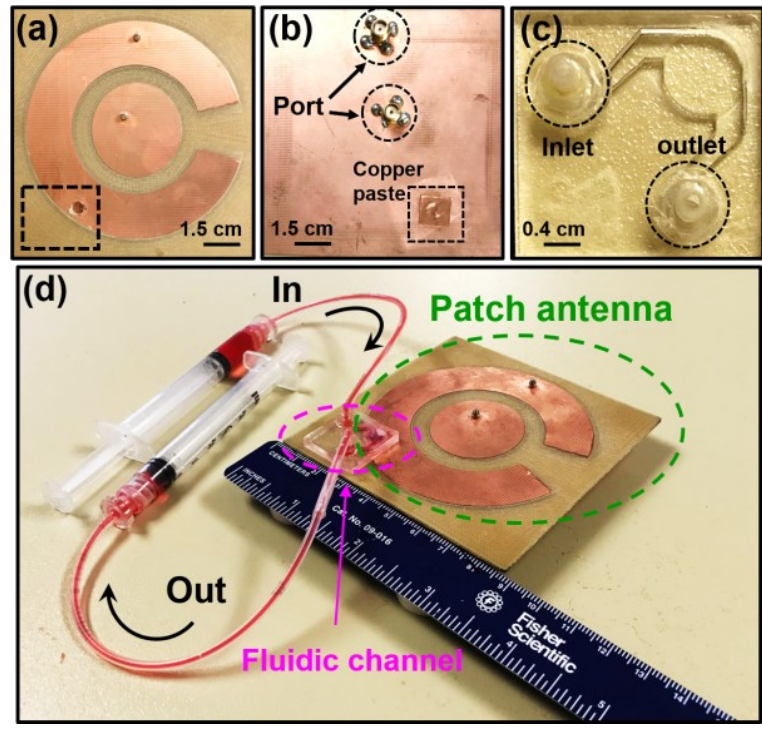

Fig. 4. Photograph of the proposed antenna: (a) Top view of the proposed dual-band patch antenna. The dashed line outlines the area of fluidic channel. (b) Bottom view of the proposed dual-band patch antenna. (c) Top view of the fluidic channel. (d) Integrated passive harmonic sensor with fluidic channel.

Fig. 1. The FHSS pattern analysis can provide robust and accurate absolute resonance sensing, which is not possible with one-dimensional data obtained from traditional non-hopping harmonic RFID sensors.

\section{Theoretical Modeling OF DUAL-BAND PATCh ANTENNA}

We have studied a compact, dual-resonance microstrip antenna composed of a concentric outer split-ring patch and an inner circular patch, which respectively resonate at $f_{0}\left(\mathrm{TM}_{310}\right.$ mode of the split-ring patch antenna) and $2 f_{0}\left(\mathrm{TM}_{110}\right.$ mode of the circular patch antenna), provided that feed points are suitably located [30]. However, both resonant modes have a narrow bandwidth. In order to perform the FHSS analysis, the inner circular patch must be replaced by the geometry that gives a wideband resonance. An elliptical patch with tailorable even and odd resonant modes could be the simplest possible design for effectively increasing the bandwidth around $2 f_{0}$. Additionally, unlike our previous work utilizing a rectangular patch with a microcavity to perturbate its $\mathrm{TM}_{010}$ mode [31], we further integrate the dual-resonance microstrip antenna with a practical fluidic channel loaded with different sample-under-tests (SUTs). Fig. 2 shows the geometry of the proposed dual-band microstrip antenna consisting of an elliptical patch, a concentric split-ring patch, and a fluidic channel integrated on top. The proposed patch layer is separated from the ground plane by the FR4 substrate with relative permittivity $\varepsilon_{r}=4.2$, thickness $h=1.6$ $\mathrm{mm}$, and loss tangent $\delta=0.015$. Given the fact that the antenna has a negligible thickness compared to operating wavelengths, resonance frequencies can be predicted by the cavity model [32]-[34]. In our case, two open cavities with concentric splitring and elliptical shapes are considered, respectively. The perfect electric conductor (PEC) boundary conditions are

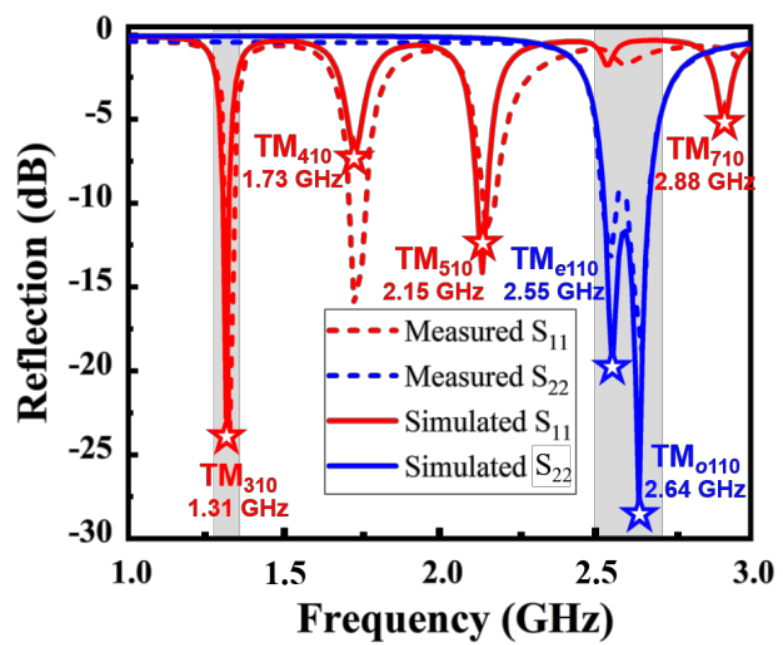

Fig. 5. Measured (dashed lines) and simulated (solid lines) reflection coefficient $\left(S_{11}\right.$ and $\left.S_{22}\right)$ for the antenna in Fig. 4. Analytical results obtained from Eqs. (1) and (2) are highlighted by stars.

applied to top and bottom layers of these two cavities, and the perfect magnetic conductor (PMC) is assumed for their sidewalls. The transcendental equation for the split-ring resonant cavity can be derived as [30]:

$$
\begin{aligned}
& J_{n}^{\prime}\left(k R_{2}\right) Y_{n}^{\prime}\left(k R_{3}\right)-J_{n}^{\prime}\left(k R_{3}\right) Y_{n}^{\prime}\left(k R_{2}\right)=0, \\
& n=\frac{m \pi}{2 \pi-\theta_{0}} \text { for } m=1,2,3 \ldots,
\end{aligned}
$$

where $J_{n}(\cdot)$ and $Y_{n}(\cdot)$ are the Bessel functions of the first and the second kinds, $k=\omega \sqrt{\varepsilon_{r} \varepsilon_{0} \mu_{0}}, \varepsilon_{0}$ and $\mu_{0}$ are the free-space permittivity and permeability. Based on Eq. (1), we have designed the split-ring patch antenna with $m=3$ ( $\mathrm{TM}_{310}$ mode), $R_{2}=38.2 \mathrm{~mm}, R_{3}=22 \mathrm{~mm}$, and $\theta_{0}=30^{\circ}$, which provides a narrow-band resonance at the fundamental frequency (1.31 $\mathrm{GHz}$ here).

For the elliptical cavity with the reference elliptical coordinate $(\xi, \eta)$, where $\xi \in[0, \infty]$ and $\eta \in[0,2 \pi]$, the transcendental equations can be reduced to the following set, with PMC boundary conditions assumed at the sidewall $\xi=\xi_{0}$ [35], [36]:

$$
\begin{array}{ll}
M_{e, n}^{\prime(1)}\left(\xi_{0}, q\right)=0 & \text { for } n \text {-th even mode } \\
M_{o, n}^{\prime(1)}\left(\xi_{0}, q\right)=0 & \text { for } n \text {-th odd mode }
\end{array}
$$

where $q=c^{2} k^{2} / 4$, the semi-focal length $c=\sqrt{a^{2}-b^{2}}$, $M_{e, n}^{(1)}(\xi, q)$ and $M_{o, n}^{(1)}(\xi, q)$ are respectively the even and odd radial Mathieu function of the first kind, $n$ is the order of the angular Mathieu functions $C_{e, n}(\eta, q)$ and $S_{e, n}(\eta, q)$, which determine the azimuthal variation along $\eta$ [37], [38]. By solving the above transcendental equation with $\xi_{0}=1.83(a=16.5 \mathrm{~mm}$, and $b=15.675 \mathrm{~mm})$, the first even-order mode $\left(\mathrm{TM}_{e 110}\right.$ at 2.55 $\mathrm{GHz})$ and the first odd-order mode $\left(\mathrm{TM}_{o 110}\right.$ at $\left.2.64 \mathrm{GHz}\right)$ can be excited in the second-harmonic band for achieving a wideband 
1

2

3

4

5

6

7

8

9

10

11

12

13

14

15

16

17

18

19

20

21

22

23

24

25

26

27

28

29

30

31

32

33

34

35

36

37

38

39

40

41

42

43

44

45

46

47

48

49

50

51

52

53

54

55

56

57
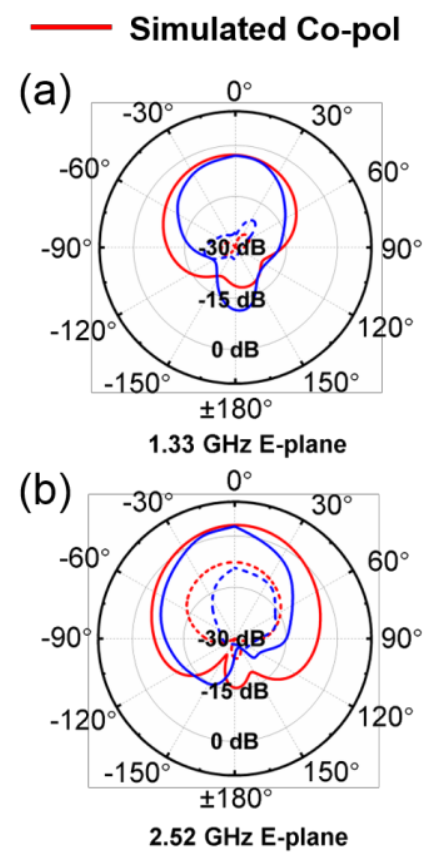

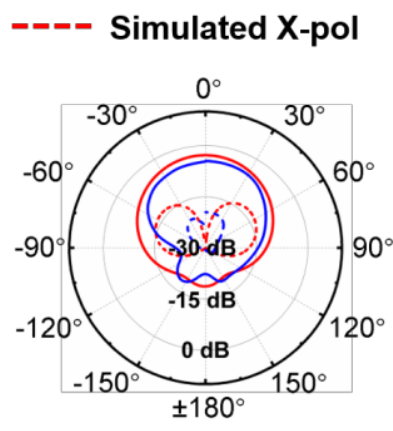

$1.33 \mathrm{GHz} \mathrm{H}$-plane

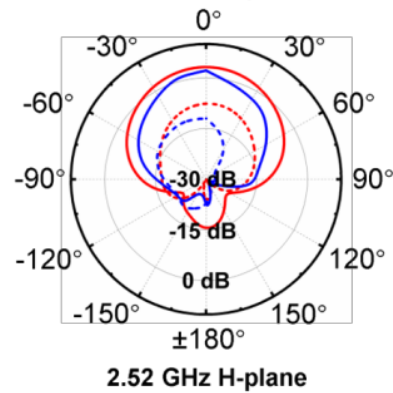

Gain in $\mathrm{dBi}$ vs. $\theta$ in degree

(d)
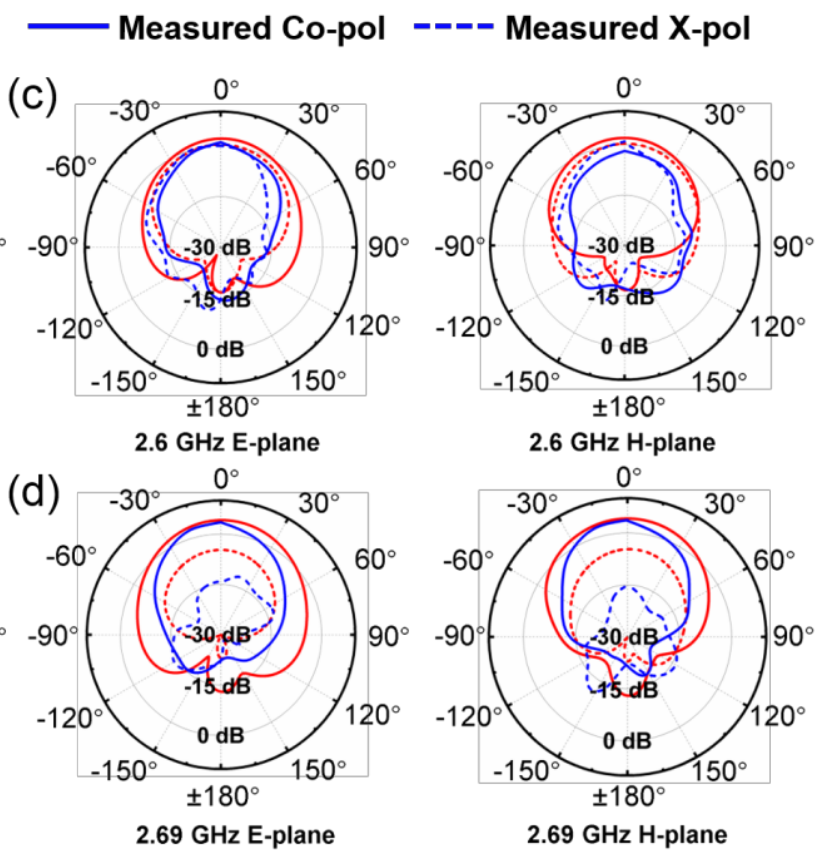

2.69 GHz H-plane

Fig. 6. Radiation patterns for the microstrip patch antenna in Fig. 4 on the E- and H-planes at: (a) $1.33 \mathrm{GHz}$, (b) $2.52 \mathrm{GHz}$, (c) $2.6 \mathrm{GHz}$, and (d) $2.69 \mathrm{GHz}$.

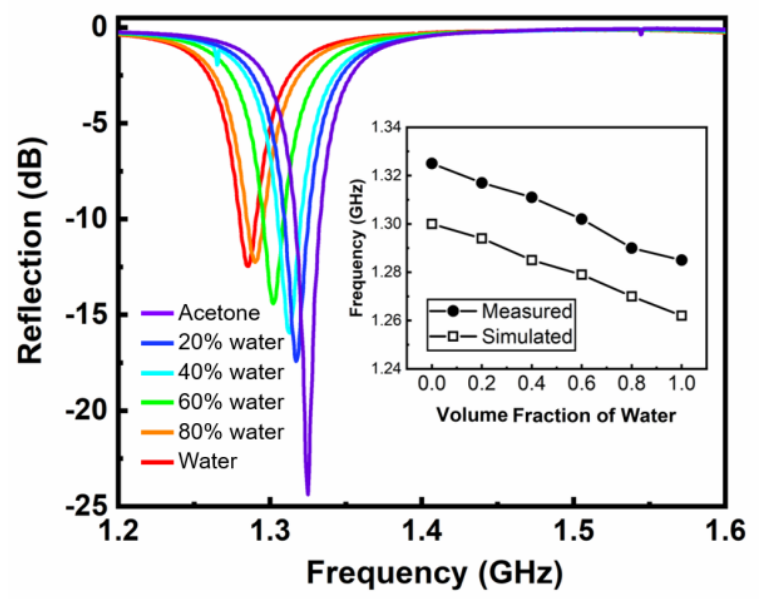

Fig. 7. Measured reflection spectrum $\left(\mathrm{TM}_{310}\right.$ mode) for the microstrip patch antenna, loaded with the acetone-water mixture at different concentrations; the inset shows the measured and calculated resonance frequency $f_{0}$ against the volume fraction of water.

Table II

Complex permittivities of different acetone-water mixtures.

\begin{tabular}{c|c|c|c|c|c|c}
\hline \hline Mixtures & $\begin{array}{c}0 \% \\
\text { (acetone) }\end{array}$ & $20 \%$ & $40 \%$ & $60 \%$ & $80 \%$ & $\begin{array}{c}100 \% \\
\text { (water) }\end{array}$ \\
\hline$\varepsilon_{r}^{\prime}$ & 20.7 & 25 & 35 & 45 & 66 & 80 \\
\hline$\varepsilon^{\prime \prime}{ }_{r}$ & 0.5 & 1 & 1.65 & 2.5 & 4.3 & 6 \\
\hline \hline
\end{tabular}

resonance. We have conducted full-wave numerical simulations [39] to validate the analytical results. Figs. 3(a)-(c) present snapshots of electric field distributions for the $\mathrm{TM}_{310}, \mathrm{TM}_{e 110}$ and $\mathrm{TM}_{0110}$ modes, respectively. The simulated field distributions are in good agreement with results obtained from

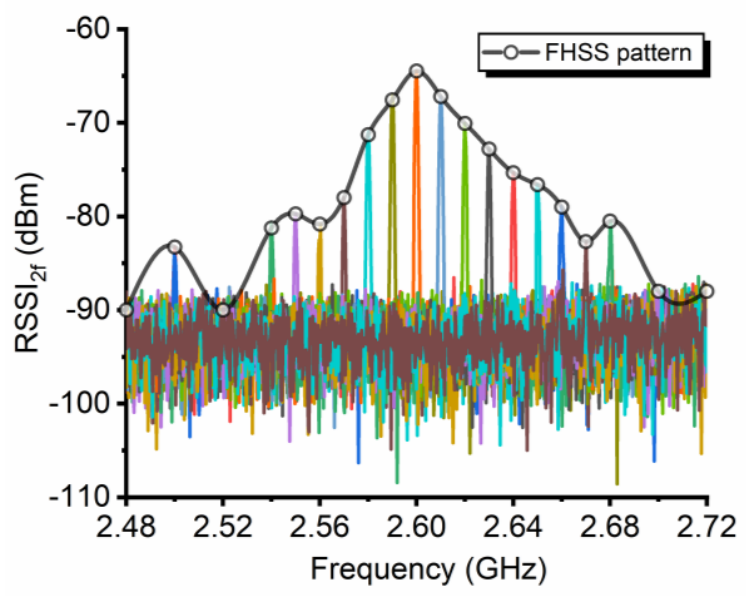

Fig. 8. Measured harmonic RSSI array for the water-acetone mixture (60 $\%$ ), which was recorded by the spectrum analyzer connected to the sniffer $R_{x}$. The envelope of this RSSI array forms the FHSS pattern that contains sensing information for the fully-passive harmonic sensor.

the cavity model, showing that the $\mathrm{TM}_{e 110}$ and $\mathrm{TM}_{o 110}$ modes exhibit orthogonal modal patterns.

\section{EXPERIMENTAL RESULTS}

According to the theoretical analysis, we have fabricated the dual-band microstrip antenna based on the FR4 substrate and copper $(\mathrm{Cu})$ microstrips, as shown in Fig. 4. The important design parameters are listed in Table I. In particular, it should be noted that a $50 \mathrm{ohms}$ coaxial cable feed is positioned at $x=$ $5 \mathrm{~mm}, y=4.5 \mathrm{~mm}$ to simultaneously excite the $\mathrm{TM}_{e 110}(2.55$ $\mathrm{GHz})$ and $\mathrm{TM}_{0110}(2.64 \mathrm{GHz})$ modes of the elliptical patch. A fluidic cavity with radius $r=2 \mathrm{~mm}$ is drilled at position $x=-27$ $\mathrm{mm}$ and $y=-20 \mathrm{~mm}$, where the $\mathrm{TM}_{310}$ resonant mode exhibits 


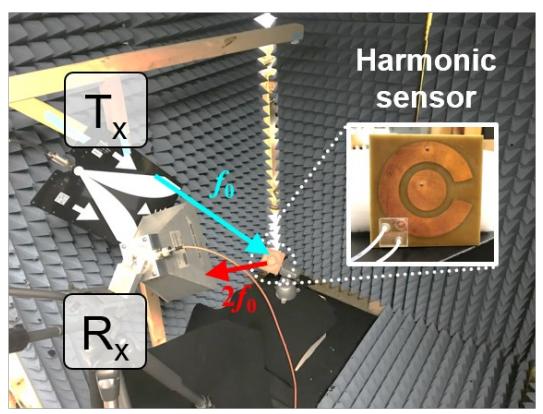

(a)

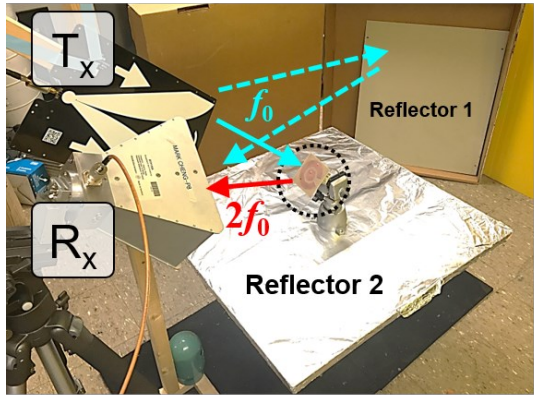

(b)

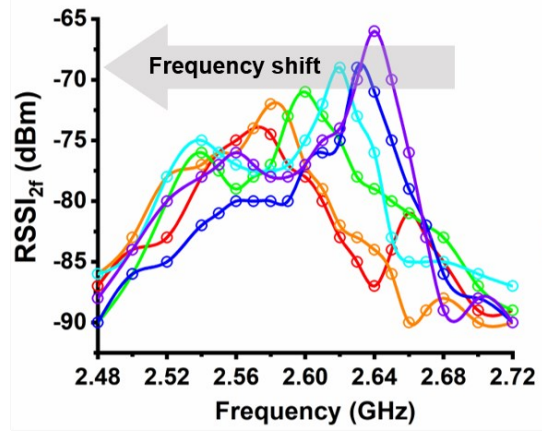

(c)

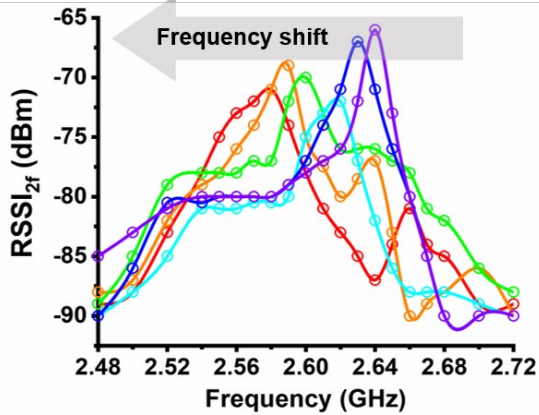

(d)

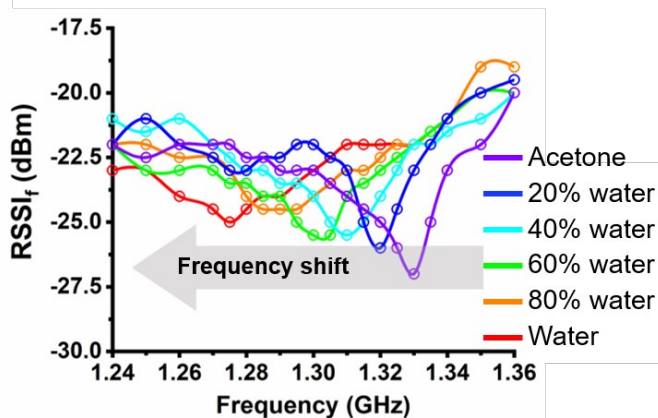

(e)

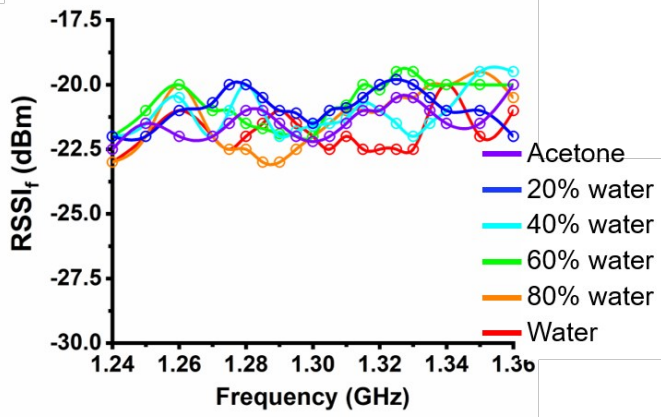

(f)

Fig. 9. Experimental setup for characterizing the wireless sensors: (a) interference-free environment (anechoic chamber) and (b) rich-scattering indoor environment. Measured FHSS pattern for the harmonic sensor in (c) interference-free and (d) rich-scattering environments. Determination of the volume fraction in acetone-water mixture is possible by tracking the peak frequency in the FHSS patterns. (e), (f) are similar to (c), (d), but for the linear backscatter sensor.

the maximum electric field strength [see Fig. 3(a)]. To accurately control the volume in the microcavity, a fluidic channel consisting of three acrylic layers with thickness $h_{1}, h_{2}$ and $h_{3}$ was fabricated [see Table I]. Each layer was etched into the specific shape [Fig. 2] by using Epilog Mini 24 laser cutter, and all layers were tied together using the double-sided adhesive tape. Subsequently, the assembled fluidic channel was mounted onto the patch antenna and connected with two plastic tubes (i.e., inlet and outlet) [Fig. 4]. Fig. 5 reports the simulated (solid lines) and measured (dashed lines) reflection coefficients versus frequency for this dual-band microstrip antenna. The resonance frequencies calculated using Eqs. (1) and (2) are also highlighted (red stars) here, which agree well with the full-wave simulation results. This measurement results confirm the dualband behavior of the antenna, with a reflection dip at $1.33 \mathrm{GHz}$ and a broadband resonance at its doubled frequency $(2.5 \mathrm{GHz}$ $2.7 \mathrm{GHz}$ ). Although the harmonic sensor is designed to work in the S-band range (2-4 GHz), the concept and design can be readily transferred to other frequency bands. Fig. 6 reports the simulated and measured radiation patterns of the proposed microstrip antenna on the E- and H-planes at the fundamental frequency and the second-harmonic band. Note that for the $\mathrm{TM}_{310}$ mode, the E-plane is $y z$-plane and the H-plane is $x z$-plane in 3D space, and those for the $\mathrm{TM}_{e 110}\left(\mathrm{TM}_{o 110}\right)$ modes are $y z$ - and $x z$ planes ( $x z-$ and $y z$-planes). The measured co-polarization radiation patterns with broadside radiation properties are in good agreement with the simulation results. At $1.33 \mathrm{GHz}\left(\mathrm{TM}_{310}\right.$ mode), this antenna exhibits a maximum measured gain of -1.5 $\mathrm{dBi}$, with a half-power beam width (HPBW) of $72^{\circ}$ on the E- plane and a HPBW of $106^{\circ}$ on H-plane. In the second-harmonic band, the $\mathrm{TM}_{e 110}$ mode $(2.52 \mathrm{GHz})$ and $\mathrm{TM}_{o 110} \operatorname{mode}(2.69 \mathrm{GHz})$ exhibit orthogonal linear polarizations, whereas circular polarizations are obtained between the two modes $(2.6 \mathrm{GHz})$. At $2.52 \mathrm{GHz}$, the antenna exhibits a maximum gain of $4.5 \mathrm{dBi}$, with a HPBW of $84^{\circ}\left(120^{\circ}\right)$ on the E-plane (H-plane). At $2.69 \mathrm{GHz}$, the maximum antenna gain is $5.7 \mathrm{dBi}$, with a HPBW of $77^{\circ}$ $\left(115^{\circ}\right)$ on the E-plane (H-plane). In these two cases, a high copolarization discrimination is apparently seen, with a crosspolarization less than $-15 \mathrm{~dB}(-10 \mathrm{~dB})$ on the E-plane (H-plane). At $2.6 \mathrm{GHz}$, the antenna shows a maximum gain of $5.3 \mathrm{dBi}$ and a HPBW of $86^{\circ}\left(116^{\circ}\right)$ on the E-plane (H-plane). In this case, the cross-polarization radiation pattern is similar to the copolarization pattern, in light of intrinsic circular polarization properties of the elliptical patch. Due to relatively high dielectric and conduction losses in the FR4 substrate, the measured radiation efficiency is $27 \%$ at $1.31 \mathrm{GHz}$ and is greater than $80 \%$ in the second-harmonic band. The realized gain of the proposed antennas can be further enhanced by selecting a high-quality substrate with minimum power dissipation.

Next, we will demonstrate the sensing function of the proposed reconfigurable dual-band antenna in terms of reflection spectrum. To this end, acetone and water mixtures with various concentrations were prepared with their complex permittivities listed in Table II [40]-[42]. Based on the perturbation theory [43], [44], the dielectric properties of the SUT filled into the fluidic cavity can be characterized by detecting the up- or down-shifting resonance frequency $f_{0}$. In this study, to track the resonant frequency variation of the dual- 


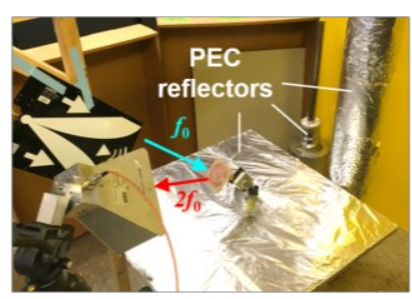

(a)

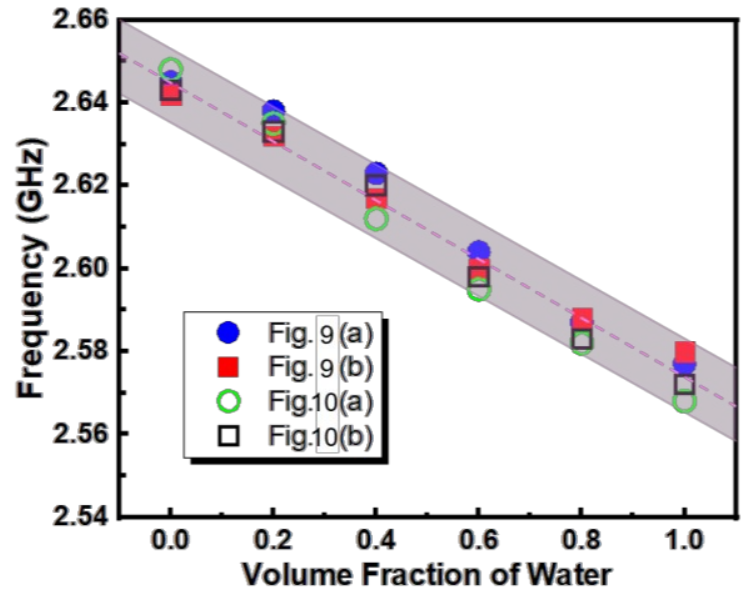

(c)

Fig. 10. Experimental setup in rich-scattering indoor environment with (a) two more metal reflectors and (b) six metal reflectors. (c) Peak frequency against the volume fraction of water in the acetone-water mixture; here, data was obtained using the harmonic sensor placed in different environments including Figs. 9(a) and 9(b).

band antenna, the Agilent N5222A PNA microwave network analyzer was used here to record the reflection spectrum of the antenna, i.e., $S_{11}$ and $S_{22}$, and the measured reflection coefficients for acetone-water mixtures of different concentrations are shown in Fig. 7. It can be clearly seen that with the increase of the volume ratio of water to acetone (effectively, increasing the liquid's dielectric constant), the resonance frequency of the $\mathrm{TM}_{310}$ mode downshifts from $1.325 \mathrm{GHz}$ to $1.287 \mathrm{GHz}$, while the second-harmonic band (coupled $\mathrm{TM}_{e 110}$ and $\mathrm{TM}_{o 110}$ modes) remains unchanged (not shown here). The inset of Fig. 7 shows the simulated and measured resonance frequency of the $\mathrm{TM}_{310}$ mode for different volume fractions of water in the mixture. It is evident that simulations and measurements are in good agreement, and both show excellent sensitivity and linearity in determination of volume fraction in binary liquid mixtures.

By connecting the aforementioned reconfigurable dual-band antenna to a passive frequency doubler [45], a fully passive wireless harmonic sensor can be built. Here, the bistatic radar measurement setup was used for the wireless sensor telemetry. The transceiver $\left(T_{x}\right)$ continuously transmits the frequencyhopped RF signal with 20 channels to the harmonic sensor, which then resends the second harmonic to the sniffer device $\left(R_{x}\right)$. The envelope of these backscattered second harmonic tones recorded by the $R_{x}$ forms the FHSS pattern, whose peak position depends on the effective dielectric constant of the loaded liquid mixtures, as shown in Fig. 8. This wireless far field sensing mechanism can be comprehensively explained as below.
According to the Friis transmission equation [46], the ratio of the power received by $R_{x}$ to the power launched by $T_{x}$ is given by:

$$
\frac{P_{r}}{P_{t}}=\left(\frac{\lambda_{0}}{4 \pi R_{1}}\right)^{2} \cdot\left(\frac{\lambda_{0} / 2}{4 \pi R_{2}}\right)^{2} \cdot \frac{G_{1} G_{2} G_{T} G_{R}}{L_{s y s}} \cdot\left|\hat{\rho}_{1} \cdot \hat{\rho}_{t}\right|^{2} \cdot\left|\hat{\rho}_{2} \cdot \hat{\rho}_{r}\right|^{2}
$$

where $G_{1}\left(G_{2}\right)$ is the realized gain of the dual-band antenna on the harmonic sensor at $f_{0}\left(2 f_{0}\right), G_{T}\left(G_{R}\right)$ denotes the realized gain of the transceiver (sniffer) used to interrogate the passive sensor, $R_{1}\left(R_{2}\right)$ is the distance between $T_{x}\left(R_{x}\right)$ and the harmonic sensor, $L_{s y s}$ is the system loss including the frequency-conversion loss and parasitics. Here, important parameters are: $P_{t}=25 \mathrm{dBm}, R_{I}$ $=R_{2}=1.0 \mathrm{~m}, G_{T}=5.5 \mathrm{dBi}$ at $1.1 \mathrm{GHz}$ and $G_{R}=12 \mathrm{dBi}$ at 2.6 GHz. In Eq. (3), $\left|\hat{\rho}_{1} \cdot \hat{\rho}_{t}\right|^{2}$ is the polarization coupling factor between the $T_{x}$ antenna and the harmonic sensor, which is approximately equal to unity if the two antennas are well aligned. On the other hand, $\left|\hat{\rho}_{2} \cdot \hat{\rho}_{r}\right|^{2}$ accounts for the polarization match between the $R_{x}$ antenna and the harmonic sensor. Since the $\mathrm{TM}_{e 110}$ mode and $\mathrm{TM}_{o 110}$ modes excited in the second-harmonic band have orthogonal polarizations [see Figs. 3 and 6], the $R_{x}$ antenna is titled by $45^{\circ}$ to capture radiation produced by both modes; here, $\left|\hat{\rho}_{2} \cdot \hat{\rho}_{r}\right|^{2}$ is $\sim 50 \%$ in the entire second-harmonic band. In summary, according to Eq. (3), the received power $P_{r}$ and RSSI are proportional to the realized gain $G_{l}$ and $G_{2}$, in which $G_{2}$ remains almost constant with a \pm 0.5 dB fluctuation, while $G_{I}$ is quite sensitive to the concentration of the acetone-water mixture. When the acetone concentration decreases, which is corresponding to the increase of the dielectric constant of the mixture, the frequency of peak $G_{l}$ is shifted from $1.325 \mathrm{GHz}$ to $1.287 \mathrm{GHz}$ [see Fig. 7], leading to the FHSS pattern downshift as can be seen in Fig. 9(c) (measured in an anechoic chamber) and Fig. 9(d) (measured in a rich-scattering indoor environment). It is evidently seen that the peak RSSI of FHSS pattern is sensitively shifted from 2.642 $\mathrm{GHz}$ to $2.58 \mathrm{GHz}$ and that such a trend is independent of the density of the electromagnetic environment. In this work, for comparison, we also employed the conventional passive backscatter sensor to wirelessly monitor the same liquid mixtures. In this case, the dual-band microstrip antenna is disconnected from the frequency doubler and its port 1 (labeled in Fig. 2(b)) is terminated by a $50 \Omega$ match load. We notice that in passive scattering events, the extinction cross section is the sum of absorption and scattering cross sections. A dip in RSSI and RCS spectrum is sometimes observed at the resonance. In this case, the incident power is absorbed without causing too much scattering; namely, the absorption cross section is greater than the scattering cross section [47]. Figs. 9(e) and 9(f) report the measured FHSS pattern for the conventional backscatter sensor placed in the anechoic chamber and the noisy environment, respectively. Although the backscatter sensor can function well in a noise-free anechoic chamber, it fails to comprehensively detect the liquid properties in rich-scattering 


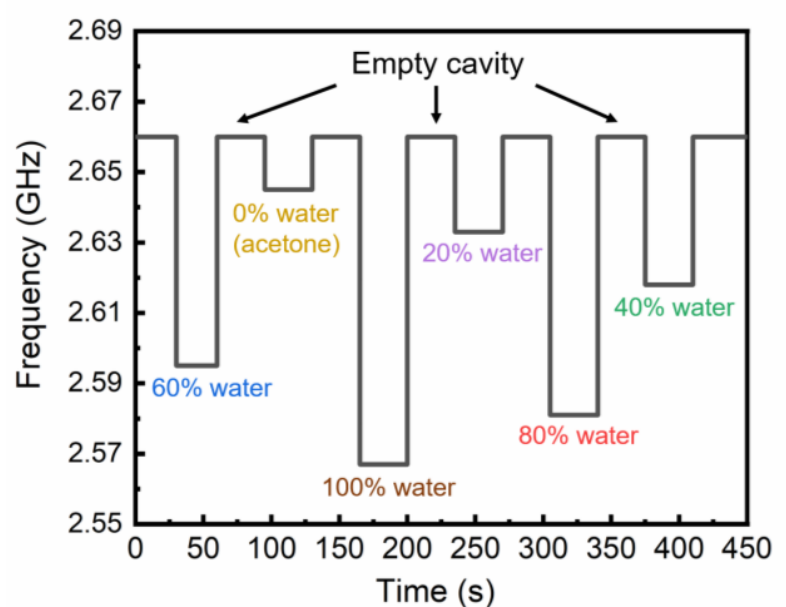

Fig. 11. Real time measurement (transient response) for the passive harmonic sensor working in a noisy environment (Fig. 10(b)); here, different acetone-water mixtures were injected in and removed from the fluidic cavity every minute, with a $30 \mathrm{~s}$ interval for the empty state reference.

environments involving echoes, clutters, multipath scattering, and the possible crosstalk between $T_{x}$ and $R_{x}$. Apparently, compared to conventional backscatter sensors, our proposed frequency-hopping harmonic sensor can robustly and sensitively detect dielectric properties of liquid mixtures, regardless of background interferences. Fig. 10 summarizes the measured peak values of FHSS pattern as a function of the water volume fraction in different scattering-rich environments. It is evident that increasing the number of large PEC scatters surrounding the harmonic sensor would not affect the sensing performance, which further demonstrates the robustness and effectiveness of our frequency-hopping harmonic sensor. Moreover, we can define our sensitivity as the slope of the curve, i.e., $-0.62 \mathrm{MHz} / 1 \%$, which means when the volume fraction of water is increased by $1 \%$, the peak of the FHSS pattern will be downshifted by $0.62 \mathrm{MHz}$. Such sensitivity and resolvability allow us to differentiate two liquids with a difference of $5 \%$ water.

Finally, we have validated the repeatability and real time capability (transient response) of this harmonic sensor working in a noisy environment. Here, different acetone-water mixtures were injected in and removed from the fluidic cavity every minute, with a $30 \mathrm{~s}$ interval for the empty state reference. The measurement results reported in Fig. 11 clarify that the sensor can always restore to its original state (with a peak RSSI at 2.66 $\mathrm{GHz}$ ) after several operating cycles. Such a robust and repeatable wireless sensing capability may enable many biological and healthcare monitoring applications, such as the wireless passive lab-on-chip platforms. We should also note that the proposed compact harmonic sensor may be integrated with more complex fluidic channels and diagnostic assays to maximize its potential for high-performance wireless sensing and telemetering.

\section{CONCLUSION}

We have proposed a low-profile, reconfigurable dual-band microstrip antenna for realizing far field wireless frequencyhopping harmonic sensors. The proposed microstrip antenna, loaded appropriately with a fluidic channel, can exhibit a tunable narrow resonance at the fundamental frequency and an invariant wideband resonance at the second-harmonic frequency. We have demonstrated that such an antenna is particularly useful for the frequency-hopping telemetry scheme, which allows for robust wireless liquid sensing in noisy environments (e.g., dense urban and indoor areas). The implementation of compact zero-power harmonic sensors with the capability of noise suppression may pave the way towards future integrated IoT sensors and batteryless smart RFID tags.

\section{REFERENCES}

[1] X. L. Jia, Q. Y. Feng, T. H. Fan, and Q. S. Lei, "RFID technology and its applications in Internet of Things (IoT)," in 2012 2nd Int. Conf. Consum. Electron, Commun. Netw, China, 2012.

[2] E. Welbourne, et al., "Building the Internet of Things using RFID: the RFID ecosystem experience." IEEE Internet Computing, vol. 13.3, pp. 48-55, 2009.

[3] G. Bedi, G. K. Venayagamoorthy, R. Singh, R. R. Brooks, K. K. Wang, "Review of Internet of Things (IoT) in electric power and energy systems," IEEE Internet Things J., vol. 5, no. 2, pp. 847-870, 2018.

[4] S. Amendola, R. Lodato, S. Manzari, C. Occhiuzzi, and G. Marrocco, "RFID technology for IoT-based personal healthcare in smart spaces," IEEE Internet Things $J$., vol. 1, no. 2, pp. 144-152, 2014.

[5] S. Preradovic, I. Balbin, N. C. Karmakar, and G. F. Swiegers, "Multiresonator-based chipless RFID system for low-cost item tracking," IEEE Trans. Microw. Theory Tech., vol. 57, no. 5, pp. 1411$1419,2009$.

[6] K. G. Ong, K. F. Zeng, and C. A. Grimes, "A wireless, passive carbon nanotube-based," IEEE Sens. J., vol. 2, no. 2, pp. 82-88, 2002.

[7] R. Bhattacharyya, C. Floerkemeier, and S. Sarma, "Low-cost, ubiquitous RFID-tag-antenna-based sensing," Proc. IEEE, vol. 98, no. 9, pp. 1593 -1600 , Sep. 2010.

[8] F. Alimenti, and L. Roselli, "Theory on zero-power RFID sensors based on harmonic generation and orthogonally polarized antennas, " Progress In Electromagnetics Research, Vol. 134, 337-357, 2013.

[9] K. Rasilainen, J. Ilvonen, A. Lehtovuori, J. M. Hannula, and V. Viikari, "Harmonic transponders: Performance and challenges," Progress In Electromagnetics Research, Vol. 134, 337-357, 2013.

[10] K. Rasilainen, J. Ilvonen, and V. Viikari, "Antenna matching at harmonic frequencies to complex load impedance," IEEE Antennas Wireless Propag. Lett., vol. 14, pp. 535-538, 2014.

[11] K. Rasilainen, J. Ilvonen, A. Lehtovuori, J. M. Hannula, V. Viikari, "On design and evaluation of harmonic transponders" IEEE Trans. Antennas Propag., vol. 63 no. 1 pp. 15-23 Jan. 2015

[12] X. Q. Guo, S. N. N, L. Guo, S. Hemour, and K. Wu, "Diplexer-based fully passive harmonic transponder for sub-6-GHz 5G-compatible IoT Applications," IEEE Trans. Microw. Theory Tech., to be published, pp. 1-3, 2019.

[13] Y. Feng, Y. Li, L. Li, B. Ma, H. Hao, and L. Li, "Tissue-dependent comatching method for dual-mode antenna in implantable neurostimulators," IEEE Trans. Antennas Propag., vol. 67, no. 8, pp. 5253-5264, Aug. 2019.

[14] Y. Li, Z. Zhang, W. Chen, Z. Feng, and M. F. Iskander, "A switchable matching circuit for compact wideband antenna designs," IEEE Trans. Antennas Propag., vol. 58, pp. 3450-3457, Nov.2010

[15] Y. Li, Z. Zhang, W. Chen, Z. Feng, and M. F. Iskander, "A compact DVB-H antenna with varactor-tuned matching circuit," Microw. Opt. Technol. Lett., vol. 52, no. 8, pp. 1786-1789, 2010. 
[16] Y. Feng, Y. Li, L. Li, B. Ma, H. Hao, and L. Li, "Design and system verification of reconfigurable matching circuits for implantable antennas in tissues with broad permittivity range," IEEE Trans. Antennas Propag., Nov.2019

[17] B. G. Colpitts, G. Boiteau, "Harmonic radar transceiver design: miniature tags for insect tracking," IEEE Trans. Antennas Propag., vol. 52, no. 11, pp. 2825-2832, 2004.

[18] D. Mascanzoni and H. Wallin, "The harmonic radar: A new method of tracing insects in the fields," Ecol. Entomol., vol. 11, pp. 387-390, 1986.

[19] J. R. Riley, A. D. Smith, D. R. Reynolds, and A. S. Edwards, "Tracking bees with harmonic radar," Nature, vol. 379, pp. 29-30, 1996.

[20] D. Psychoudakis, W. Moulder, C.-C. Chen, H. Zhu, and J. L. Volakis, "A portable low-power harmonic radar system and conformal tag for insect tracking, "IEEE Antennas Wireless Propag. Lett., vol. 7, pp. 444447, 2008.

[21] J. L. Osborne et al., "A landscape-scale study of bumble bee foraging range and constancy using harmonic radar," J. Appl. Ecol., vol. 36, pp. 519-533, Sep. 1999.

[22] C. Van Tilburg et al., "Wilderness Medical Society practice guidelines for prevention and management of avalanche and nonavalanche snow burial accidents," Wilderness Environ. Med., vol. 28, no. 1, pp. 23-42, Mar. 2017.

[23] M. Hajizadegan, M. Sakhdari, L. Zhu, Q. Cui, H. Huang, M. C. Cheng, and P. Y. Chen, "Graphene sensing modulator: Toward low-noise, selfpowered wireless microsensors," IEEE Sens. J., vol. 17, no. 22, pp. 72397247, 2017.

[24] H. Huang, P. Y. Chen, C. H. Hung, R. Gharpurey, and D. Akinwande, "A zero power harmonic transponder sensor for ubiquitous wireless $\mu \mathrm{L}$ liquid-volume monitoring," Sci. Rep., vol. 6, p. 18795, 2016.

[25] H. Huang, M. Sakhdari, M. Hajizadegan, A. Shahini, D. Akinwande, and P. Y. Chen, "Toward transparent and self-activated graphene harmonic transponder sensors," Appl. Phys. Lett., vol. 108, no. 17, p. 173503, 2016.

[26] A. Lazaro, R. Villarino, and D. Girbau, "A Passive Harmonic Tag for Humidity Sensing," International Journal of Antennas and Propagation, vol. 2014, Article ID 670345, 11 pages, 2014.

[27] H. Huang, L. Tao, F. Liu, et al, "Chemical sensitive graphene modulator with a memory effect for internet-of-things applications," Microsyst. Nanoeng., vol. 2, p. 16018, 2016.

[28] D. Ahbe, S. Beer, T. Zwick, Y. Wang, and M. M. Tentzeris, "Dual-band antennas for frequency-doubler-based wireless strain sensing," IEEE Antennas Wireless Propag. Lett., vol. 11, pp. 216-219, 2012.

[29] C. Cho, X. H. Yi, D. Li, Y. Wang, and M. M. Tentzeris, "Passive wireless Frequency doubling antenna sensor for strain and crack sensing," IEEE Sens. J, vol. 16, no. 14, pp. 5725-5733, 2016.

[30] L. Zhu, N. Alkhaldi, H. M. Kadry, S. L. Liao, and P. Y. Chen, "A compact hybrid-fed microstrip antenna for harmonics-based radar and sensor system," IEEE Antennas Wireless Propag. Lett., vol. 17, no. 12, pp. 2444-2448, 2018.

[31] L. Zhu, and P. Y. Chen, "A compact, zero-power and low-noise harmonic-transponder for liquid and moisture sensing," in 2019 IEEE International Symposium on Antennas and Propagation and USNCURSI Radio Science Meeting, Atlanta, GA, USA, USA, 2019.

[32] K. R. Carver and J. W. Min, "Microstrip antenna technolog," IEEE Trans. Antennas Propag., vol. 29, no. 1, pp. 2-24, 1981.

[33] W. F. Richards, Y. T. Lo, and D. D. Harriso, "An improved theory of microstrip antennas with applications," IEEE Trans. Antennas Propag., vol. 29, no. 1, pp. 34-46, 1981.

[34] D. M. Pozar, Microwave Engineering, 4th Edition, John Wiley \& Sons, 2012.

[35] J. C. Gutierrez-vega, R. M. Rodriguez-Dagnino, M. A. Meneses-Nava, and S. Chavez-Cerda, "Mathieu functions, a visual approach," Am. J. Phys., vol. 71, no. 3, pp. 233-242, 2003.

[36] D. A. Goldberg, L. J. Laslett and R. A. Rimmer, "Modes of elliptical waveguides: a correction," IEEE Trans. Microw. Theory Tech., vol. 38, no. 11, pp. 1603-1608, 1990.
[37] P. Y. Chen and A. Alu, "Dual-mode miniaturized elliptical patch antenna with $\mu$-negative metamaterials," IEEE Antennas Wireless Propag. Lett., vol. 9 , pp. 351 - 354, 2010.

[38] P. Y. Chen and A. Alu, "Sub-wavelength elliptical patch antenna loaded with $\mu$-negative metamaterials," IEEE Trans. Antennas Propag., vol. 58 no. 9, pp. 2909-2919, 2010.

[39] ANSYS $®$ Academic Research Electromagnetics Suite, Release 19.0.

[40] C. Malmberg and A. Maryott, "Dielectric constant of water from 0" to $100^{\circ}$ C," J. Res. Nat. Bureau of Standards, vol. 56, pp. 1-8, 1956.

[41] G. Akerlöf, "Dielectric constants of some organic solvent-water mixtures at various temperatures," J. Am. Chem. Soc., vol. 54, p. 4125, 1932.

[42] C. Gao, T. Wei, F. Duewer, Y. Lu,and X. D. Xiang, "High spatial resolution quantitative microwave impedance microscopy by a scanning tip microwave near-field microscope," Appl. Phys. Lett., vol. 71, pp. 1872-1874, 1997.

[43] K. Saeed, R. D. Pollard, and I. C. Hunter, "Substrate integrated waveguide cavity resonators for complex permittivity characterization of materials," IEEE Trans. Microw. Theory Tech., vol. 56, no. 10, pp. 2340-2347, 2008.

[44] H. Huang, P. S. Zhao, P. Y. Chen, Y. Ren, X. Liu, M. Ferrari, Y. Hu, and D. Akinwande, "RFID tag helix antenna sensors for wireless drug dosage monitoring, " IEEE J. Transl. Eng. Health Med., vol. 2, pp. 1-8, 2014.

[45] FK-3000+ from Mini-Circuit. Availabe: www.minicircuits.com /WebStore/dashboard_html?model=FK-3000\%2B.

[46] J. A. Shaw, "Radiometry and the Friis transmission equation," Am. J. Phys., vol. 81, no. 1, pp. 33-37, 2013.

[47] A. M. J. Marindra and G. Y. Tian, "Chipless RFID sensor tag for metal crack detection and characterization," IEEE Trans. Microw. Theory Techn., vol. 66, no. 5, pp. 2452-2462, May 2018. 